\title{
$B$ cell therapy for rheumatoid arthritis: the rituximab (anti-CD20) experience
}

\author{
T Shaw, J Quan, M C Totoritis
}

Ann Rheum Dis 2003;62(Suppl II):ii55-ii59

$\mathrm{R}^{\mathrm{h}}$ heumatoid arthritis (RA) is a complex condition that is well characterised by chronic inflammation in the synovial membrane of affected joints, often with systemic manifestations. Yet the aetiology and precise pathogenesis of $\mathrm{RA}$ remain unclear. It has been postulated that there are multiple exogenous and/or endogenous antigenic triggers, which act on a predisposed genetic background to initiate a self perpetuating series of autoimmune responses in the synovial compartment, which are expressed as the signs and symptoms of RA.

The traditional principles of the treatment of RA are empirical. The use of analgesics and non-steroidal antiinflammatory drugs (NSAIDs) for the control of pain and inflammation is combined with disease modifying antirheumatic drug (DMARD) treatment to slow the processes that result in joint destruction in RA, shown by radiological changes, anatomical deformities, and joint dysfunction. The conventional mainstays of DMARD treatment include antimalarial drugs, sulfasalazine, and methotrexate.

To elucidate the aetiology and pathogenesis of RA much attention has been focused on the putative effects on the continuing inflammatory process of different synovial cell populations, including macrophages, fibroblasts, endothelial cells, $\mathrm{T}$ cells, and B cells, ${ }^{1}$ and on the influence of numerous cytokines. Studies have led to the development and introduction into clinical practice of treatments directed towards limiting these inflammatory driving effects. Such treatments include anti-tumour necrosis factor $\alpha$ (anti-TNF $\alpha)$ drugs (etanercept, infliximab, and adalimumab) and interleukin based treatments (anakinra). Although these treatments have been shown to be very effective, they do not work in all patients. In recent years there has been growing interest in, and enhanced understanding of, the contribution of B cells to the immunopathogenesis of RA. This article will briefly review the potential role of B cells in RA and the current evidence that B cell therapy is a promising approach in its treatment.

\section{RATIONALE FOR TARGETED B CELL THERAPIES IN \\ RA}

The precise contribution of B cells to the immunopathogenesis of RA is not well characterised, although a number of researchers and commentators have suggested several mechanisms in which B cell involvement is implicated. ${ }^{2-8}$ Figure 1 and the points listed below summarise the possible mechanisms by which B cells may play a part in the disease process.

- B cells may function as antigen presenting cells and provide important costimulatory signals required for CD4 $+\mathrm{T}$ cell clonal expansion and effector functions.

- B cells in RA synovial membrane may also secrete proinflammatory cytokines such as TNF $\alpha$ and chemokines.

- Rheumatoid synovial membrane contains an abundance of B cells that produce the rheumatoid factor (RF) antibody. ${ }^{9}$ RF positive (seropositive) RA is associated with more aggressive articular disease, a higher prevalence of extraarticular manifestations, and increased morbidity and

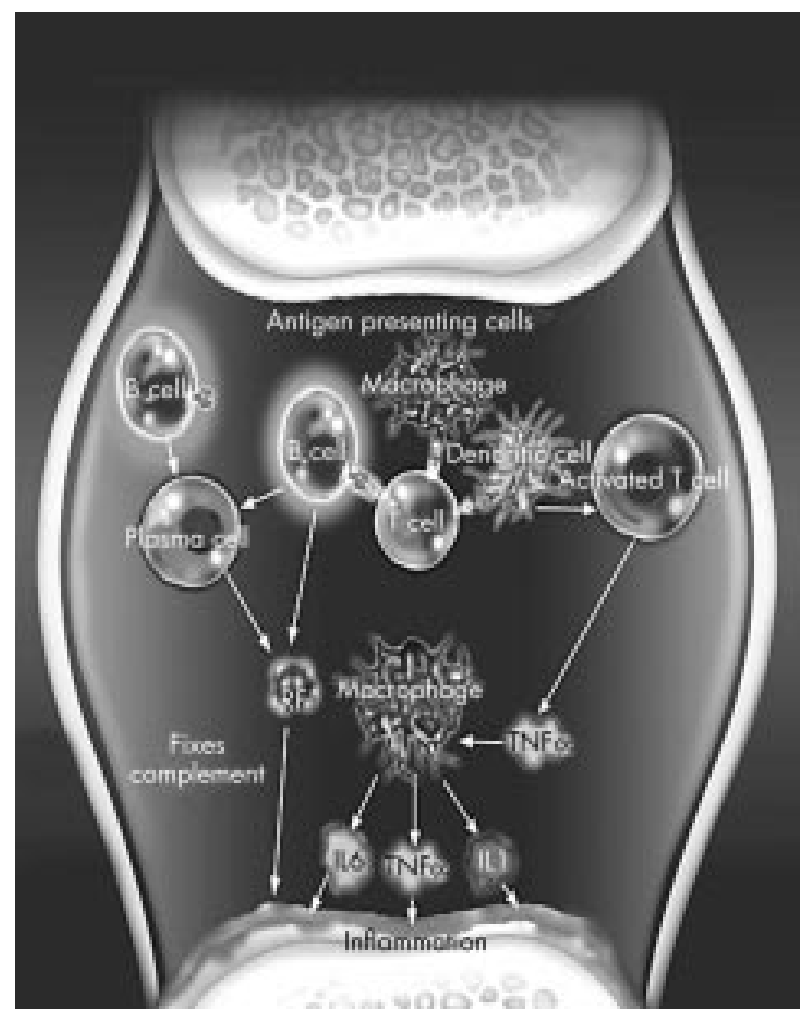

Figure 1 Potential roles of B cells in RA.

mortality. ${ }^{10}$ RF may also be a self perpetuating stimulus for B cells, potentially leading to activation and antigen presentation to Th cells, which may be mechanistically responsible for further RF production. Thus, RF immune complex mediated complement activation, in conjunction with binding of the Fc $\gamma$ receptor, collectively contribute to the propagation of the inflammatory cascade. ${ }^{2}$

- T cell activation is considered to be a key component of the pathogenesis of RA. Recent evidence indicates that this activation is critically dependent on the presence of $\mathrm{B}$ cells. $^{5}$

Although the relative importance of each of these is unknown, it is becoming increasingly clear that through multiple mechanisms B cells potentially have a central role in this

Abbreviations: ACR, American College of Rheumatology; AEs, adverse effects; DMARDs, disease modifying antirheumatic drugs; $\mathrm{NCl}-\mathrm{CTC}$,

National Cancer Institute Common Toxicity Criteria; NHL, non-Hodgkin's lymphoma; NSAIDs, non-steroidal anti-inflammatory drugs; RA,

rheumatoid arthritis; RF, rheumatoid factor; $T N F \alpha$, tumour necrosis factor $\alpha ; \mathrm{IV}$, intravenous 
disease. Thus B cells are an appropriate target for therapeutic intervention.

\section{Rituximab as a targeted B cell therapy}

Rituximab (Rituxan, MabThera) is a genetically engineered chimeric anti-CD20 monoclonal antibody. It comprises human IgGIFcK constant regions and small variable light and heavy chain regions from the anti-CD20 murine antibody fragment, IDEC-2B8, which is reactive to human CD20. CD20 is a pan-B cell surface antigenic phosphoprotein that is restricted in its expression to pre-B and mature B cells. CD20 is not present on stem cells and is lost before differentiation into plasma cells. It does not rapidly modulate upon binding anti-CD20 antibodies. From studies conducted in patients with non-Hodgkin's lymphoma (NHL), it has been shown that treatment with rituximab results in a transient depletion of selective (CD20+) $\mathrm{B}$ cells that lasts for up to six months. Thereafter, B cell levels return to normal within 9-12 months. ${ }^{11}$

Rituximab has three putative components to its mechanism of action that contribute to its B cell depletion properties.

- Antibody dependent cell mediated cytotoxicity, in which natural killer cells, macrophages, and monocytes are recruited through their $\mathrm{Fc} \gamma$ receptors bound to surface CD20. This induces CD20+ B cell lysis. ${ }^{12}$

- Complement dependent cytotoxicity induced by complexed rituximab bound to surface CD20 and binding Clq. This results in activation of the complement cascade and generation of the membrane attack complex, causing CD20+ B cell lysis. ${ }^{14}$

- Promotion of CD20+ B cell apoptosis.

\section{EXPERIENCE WITH RITUXIMAB IN NON-HODGKIN'S LYMPHOMA}

Rituximab is the first monoclonal antibody to be widely approved and marketed for the treatment of cancer. It is approved in more than 75 countries for the treatment of CD20 + B cell NHL at a standard dose regimen of $375 \mathrm{mg} / \mathrm{m}^{2}$ given as intravenous (IV) infusions every four or eight weeks. Since its market authorisation in 1997, it is estimated that over 300000 patient exposures have occurred and consequently the safety profile of rituximab within patients with NHL is well characterised.

The vast majority of adverse events (AEs) reported with rituximab are associated with infusions. They occur more commonly during the first infusion and decline with subsequent infusions. ${ }^{15}$ These generally occur within 30-120 minutes and are characterised by fever, chills or rigors, nausea, headache, and transient hypotension. Seventy to $80 \%$ of patients with NHL experience an infusion reaction during their first infusion, and this declines to about 30\% with subsequent infusions. Such events are predominantly mild to moderate in severity (grade 1 or 2 according to the National Cancer Institute Common Toxicity Criteria) (NCI-CTC) and brief. Events gradually resolve when the infusion is either slowed or briefly interrupted. Emerging evidence indicates that the overall incidence of AEs related to infusion may be lower in patients with autoimmune disease, and this will be discussed later in this article.

The large patient exposure has also permitted the identification of a number of rare events and risk factors, some of which are specific to patients with lymphoma, such as tumour lysis syndrome. Other rare events include fatal infusion reactions and severe mucocutaneous reactions (including paraneoplastic pemphigus - a rare disorder found primarily in haematological malignancies, Stevens-Johnson syndrome, and/or toxic epidermal necrolysis). Identified risk factors for tumour lysis syndrome include high numbers of circulating malignant cells $\left(>25000 / \mathrm{mm}^{3}\right)$ or high tumour burden, and for fatal infusion reactions include concomitant cardiopulmonary disease and the use of higher doses in chronic lymphocytic leukaemia or mantle cell lymphoma.
Through education and appropriate patient selection based on these risk factors, the incidence of these events is extremely low $(<1: 10000)$. Such risk factors (for example, bulky disease) are also mainly associated with patients with NHL and so are less relevant in patients with RA and other diseases.

Long term follow up with multiple courses of treatment with rituximab in NHL is currently being investigated in a number of large, multicentre, controlled studies. These should provide further information on the effect of sustained B cell depletion. In a report of a group of 36 patients with NHL who had received four courses of treatment at six month intervals, no cumulative or additional safety concerns were identified during the maintenance treatment period. ${ }^{16}$ It is important to note that there were no opportunistic infections or evidence that the overall infection rate was increased in these patients, even though they might have been B cell depleted throughout the two year study period.

\section{CLINICAL EXPERIENCE WITH RITUXIMAB IN RA Early case reports and open label study}

Early case reports of the remission of coexisting RA in patients with NHL treated with rituximab ${ }^{17}$ provided anecdotal hints that rituximab may be a clinically relevant intervention in RA. A subsequent small scale exploratory open label study ${ }^{18}$ provided the first objective indication of the therapeutic use of rituximab in RA.

In this study, ${ }^{18}$ five patients who met the American College of Rheumatology (ACR) criteria for classical RA and whose disease was inadequately controlled despite at least five previous DMARDs given sequentially as single agents, were treated with a regimen that included rituximab. After discontinuation of all previous DMARDs, and provision of analgesic treatment with diclofenac and/or co-proxamol as required, rituximab treatment was given by IV infusion. Up to four infusions were given over 22 days: $300 \mathrm{mg}$ on day 2 and $600 \mathrm{mg}$ on days 8,15 , and 22. Cyclophosphamide $750 \mathrm{mg}$ was also given by IV infusion on days 4 and 17, and patients received oral prednisolone (30-60 mg/day) for up to 22 days. This regimen was derived from three of the components of the R-CHOP regimen (rituximab, cyclophosphamide, hydroxydoxorubicin, vincristine, and prednisolone), which has produced good results in NHL. ${ }^{19}$ The use of hydroxydoxorubicin and vincristine, however, was excluded on the grounds that it is difficult to justify the use of these drugs in the treatment of RA. Patients were regularly followed up for up to 76 weeks and efficacy and safety outcomes were recorded.

All five patients showed a rapid improvement in synovitis and a substantial improvement in their swollen joint count (fig 2A) as well as other efficacy outcomes. When these outcome measures were applied to the ACR criteria for response, all patients achieved an ACR50 level of response by six months and three of these reached an ACR70 level by this time (fig 2A). Beyond six months, patients maintained their improvement or improved further. Normalisation of RF at one year occurred in two patients. Two patients who required re-treatment (one at week 34 and one at week 49 from the first infusion) ultimately also achieved ACR70 responses.

No major AEs attributable to treatment were seen and even though the B cell levels fell to undetectable levels (an expected effect of rituximab) in each of the five cases and remained low for at least six months, immunoglobulin IgG, IgA, and IgM levels showed a modest decline, but remained within normal levels. In two patients, B cell repopulation occurred without relapse and in another two patients the return of B cells coincided with the need for re-treatment. One patient was lost to follow up after six months but apparently remained well.

Although these are very positive results, the interpretation of the role of rituximab in the treatment of RA from the results of this study is confounded by the concomitant use of 
A Rituximab plus cyclophosphamide and prednisolone ${ }^{18}$

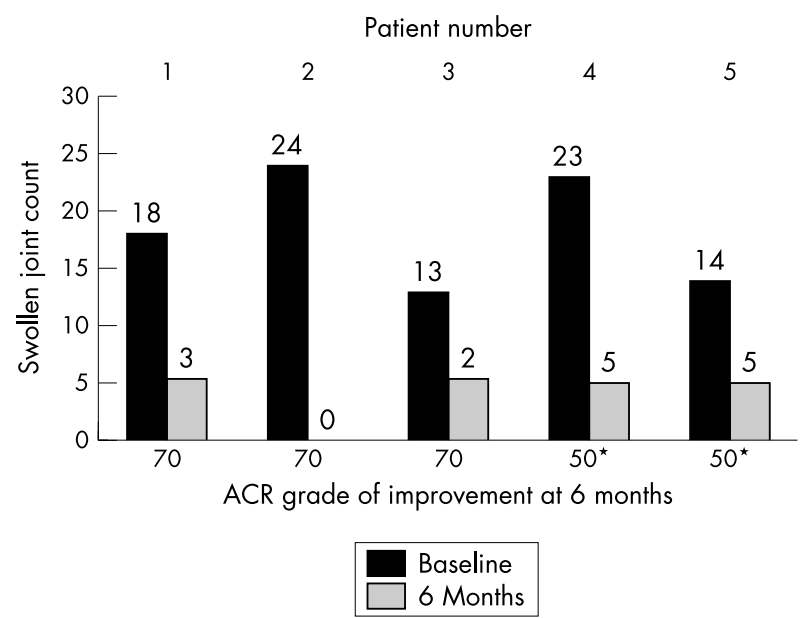

B Rituximab alone ${ }^{21}$

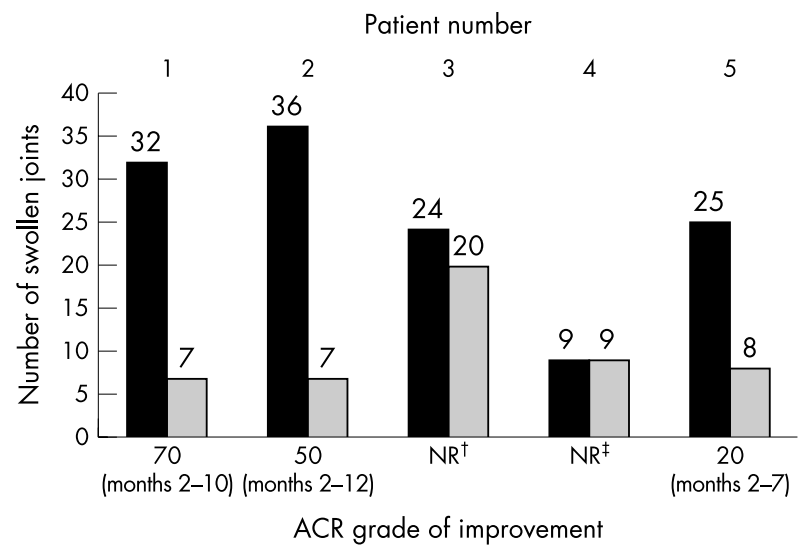

Figure 2 Response to rituximab at six months in two independent studies each involving five patients with RA refractory to DMARD treatment treated $(A)$ with or $(B)$ without concomitant DMARD treatment. $^{1821}$

cyclophosphamide and high dose prednisolone. A more robust randomised controlled double blind trial was therefore conducted to confirm the efficacy and tolerability of rituximab in RA and to explore the role of concomitant cyclophosphamide.

\section{Randomised, controlled, double blind evaluation of rituximab in RA}

In this randomised controlled trial a total of 161 patients with RA were recruited. All had active disease, were RF positive and were refractory to previous DMARD treatment despite the use of concomitant methotrexate. Patients were randomly allocated to one of four treatment groups:

- Group A: Continuation of methotrexate alone

- Group B: Rituximab alone (given as two $\times 1$ g IV infusions)

- Group C: Rituximab (two $\times$ l g IV infusions) plus cyclophosphamide (two $\times 750 \mathrm{mg}$ infusions)

- Group D: Rituximab (two $\times 1$ g IV infusions) plus continuing methotrexate.

Blinding of the trial was maintained using the double dummy technique in groups $\mathrm{A}$ and $\mathrm{B}$, and all groups also received a 17 day course of corticosteroids (total dose $910 \mathrm{mg}$ ) from the start of randomised treatment. Data from an interim cohort of the first 122 patients, followed up for 24 weeks, have been reported. ${ }^{20}$ These interim cohort data (fig 3) confirm the

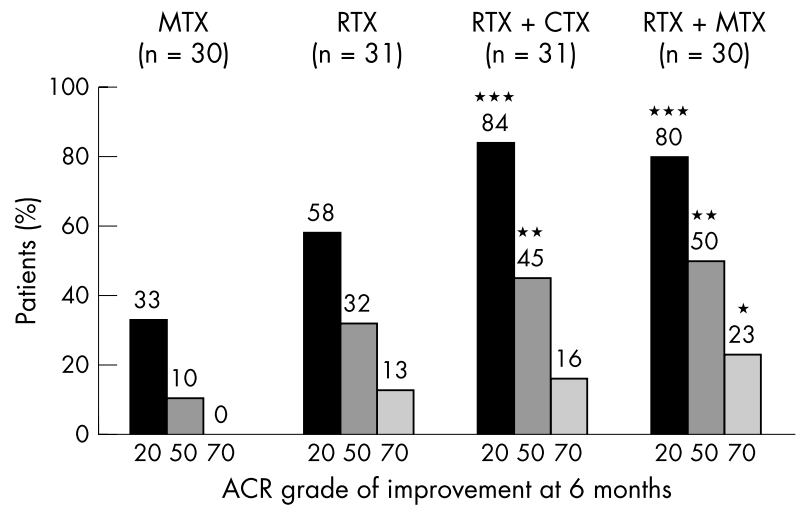

Figure 3 ACR responses at six months in an interim cohort of patients with RA refractory to DMARD treatment treated with rituximab with and without concomitant cyclophosphamide or methotrexate, compared with continuation of methotrexate alone.

early open label observations that a short induction treatment with rituximab is associated with a significant clinical benefit. Combination of rituximab with methotrexate or cyclophosphamide produced the highest levels of response with no apparent difference between the groups. The data also indicate that cyclophosphamide is not pivotal to the efficacy of rituximab, and that rituximab given as an adjunct to continuing methotrexate is an effective option.

Safety evaluations from this interim cohort show that rituximab was generally well tolerated in these patients with RA. There were no serious AEs associated with infusions of rituximab. Most reported AEs were associated with infusions and were consistent with the presence of NHL (fever, chills, rash, hypotension, predominantly of NCI-CTC grades 1 or 2 ). However, the proportion of patients with RA experiencing infusion related AEs was substantially lower (39\%) than the historical NHL comparisons $(70-80 \%)$. Possible explanations for this improved profile in patients with RA may be related to the absence of a large burden of malignant CD20 + B cells and resultant cell lysis in RA compared with NHL or to pretreatment with corticosteroids.

Eleven serious events were reported from the cohort during the 24 week study, which were generally evenly balanced across the four treatment groups. Four serious infections occurred, one of which was in the methotrexate alone group (group A). Two cases of bronchopneumonia (one of which had a fatal outcome) occurred in the rituximab groups (B and C), although the fatality was considered unrelated to rituximab by the investigator primarily because of the patient's underlying ischaemic heart disease and lack of a confirmed diagnosis of pneumonia. Overall, the three rituximab regimens were well tolerated.

The interim cohort data from this phase II trial show that a short induction regimen with rituximab alone, or in combination with either methotrexate or cyclophosphamide, produced substantial clinical benefit in patients with refractory RA. The full cohort data will be reported in due course.

\section{Additional supportive data}

The results from three subsequent independent open studies ${ }^{21-23}$ concur with the original observations and the double blind randomised study findings.

In one of these, ${ }^{21}$ five patients with active RA who met the ACR diagnostic criteria, and who were unresponsive to prior treatment that included anti-TNF $\alpha$ therapy, were treated with rituximab (375 $\mathrm{mg} / \mathrm{m}^{2}$ by IV infusion) weekly for four weeks. Unlike in the original open series, ${ }^{18}$ concomitant cyclophosphamide and high dose steroids were not given, although patients were permitted to take low dose prednisolone (5-7 
mg/day), and NSAIDs and/or antimalarial drugs concomitantly as required. At six months, three patients had achieved an ACR20 level of response and one further patient achieved an ACR20 response between months 3 and 5. In addition, there was a marked and sustained clinical improvement in two of these patients who achieved ACR70 and ACR50 levels of response, respectively (fig $2 \mathrm{~B}$ ).

Changes in B cell, RF, and IgA, IgG, and IgM levels in this series were consistent with those seen in the previous open series, ${ }^{18}$ with no serious or lasting sequelae. Although caution should be applied when comparing two open label series in this way, not least because the disease activity baselines might have been different (fig $2 \mathrm{~A} v$ fig $2 \mathrm{~B}$ ), the latter study $\mathrm{y}^{21}$ provides further evidence that B cells may have a role in the pathogenesis of RA, and that rituximab rather than cyclophosphamide and/or prednisolone drives this response, at least in a subset of patients.

That this is the case is supported by two further open label studies in patients with refractory RA. ${ }^{22}{ }^{23}$ In a 22 patient cohort that included the five patients reported by Edwards and Cambridge (2001), ${ }^{22}$ rituximab at total doses ranging between 300 and $1400 \mathrm{mg} / \mathrm{m}^{2}$, with or without cyclophosphamide and/or high dose prednisolone, clinically important responses were seen with rituximab both alone and in combination with cyclophosphamide and/or steroids when the total dose of rituximab was $>600 \mathrm{mg} / \mathrm{m}^{2}$. This observation supports the premise that cyclophosphamide and high dose steroids are unlikely to be solely responsible for achieving the high level of response seen with the combination.

An interim report of another small series of seven evaluable patients with RA refractory to DMARDs and infliximab ${ }^{23}$ lends further support to the hypothesis that $\mathrm{B}$ cells mediate the pathology of RA and that rituximab is a promising agent for the treatment of RA. These patients were treated with rituximab ( $100 \mathrm{mg}$ IV at week $1,375 \mathrm{mg} / \mathrm{m}^{2}$ at week 2 , and $500 \mathrm{mg} / \mathrm{m}^{2}$ at weeks 3 and 4 ) and followed up for a median of five months. All seven patients had improved joint scores and three of them met the criteria for an ACR20 level of response. Absolute B cell levels were diminished by eight weeks after the first infusion, with no significant changes in absolute $\mathrm{T}$ cell levels. In both studies, rituximab therapy was well tolerated and no major AEs attributable to treatment occurred. In a minority of patients who experienced transient infusion related reactions the severity was mild to moderate.

\section{RITUXIMAB IN OTHER AUTOIMMUNE CONDITIONS}

A number of reports have also cited the benefit of rituximab treatment in other autoimmune conditions such as idiopathic thrombocytopenic purpura, ${ }^{24}$ type II mixed cryoglobulinaemia ${ }^{25}$ and $\operatorname{IgM}$ antibody associated neuropathies. ${ }^{26}$ In particular, the suggestion that enhanced B cell function is the defining pathogenic event in systemic lupus erythematosus ${ }^{27}$ has been mirrored by some encouraging preliminary results with rituximab treatment in this condition. ${ }^{28}{ }^{29}$ Further definition of the potential therapeutic benefit of rituximab in these and other autoimmune conditions is continuing. ${ }^{30}$

\section{SUMMARY AND CONCLUSIONS}

The clinical data to date strongly support the premise that $\mathrm{B}$ cells play a major part in the pathogenesis of RA and that selective targeting of these cells may provide treatments that not only enhance our understanding of this disease but also provide substantial and prolonged clinical benefit. Rituximab (Rituxan, MabThera) is an anti-CD20 chimeric monoclonal antibody that produces a high level of sustained efficacy in RA that coincides with a profound and longlasting peripheral depletion of CD20+ B cells after a single treatment course. Market experience from over 300000 exposures to rituximab in patients with NHL demonstrates a well characterised safety profile. Early exposure data in RA suggest that the tolerability and safety profile of rituximab may be even better in these patients. Preliminary reports have also suggested that B cell targeting with rituximab is a promising approach for some other autoimmune conditions, particularly systemic lupus erythematosus. Further clinical trials with rituximab are underway to increase knowledge about, and further characterise the encouraging therapeutic profile of, this monoclonal antibody in RA and other autoimmune conditions.

\section{Authors' affiliations}

T Shaw, Roche Products Ltd, Welwyn Garden City, UK

J Quan, Genentech Inc, I DNA Way, South San Francisco, CA, USA

M C Totoritis, IDEC Pharmaceuticals Corp, 3030 Callan Road, San

Diego, CA, USA

Correspondence to: Mr T Shaw, Roche Products Ltd, 40 Broadwater Road, Welwyn Garden City, Hertfordshire AL7 3AY, UK;

tim.shaw@roche.com

\section{REFERENCES}

1 Panayi GS. The immunopathogenesis of rheumatoid arthritis. $\mathrm{Br} \mathrm{J}$ Rheumatol 1993;32:4-14.

2 Edwards JCW, Cambridge G, Abrahams VM. Do self-perpetuating B lymphocytes drive human autoimmune disease? Immunology 1999;97:188-196

3 Gause A, Berek C. The role of B cells in the pathogenesis of rheumatoid arthritis. Potential implications for treatment. BioDrugs 2001;15:73-79.

4 Reparon-Schuiit CC, van Esche WJE, van Kooten C, Ezendam NP, Levarht EW, Breedveld FC, et al. Presence of a population of CD20+, CD38- B lymphocytes with defective proliferative responsiveness in the synovial compartment of patients with rheumatoid arthritis. Arthritis Rheum 2001;44:2029-37

5 Takemura S, Klimiuk PA, Braun A, Goronzy JJ, Weyand CM. T cell activation in rheumatoid synovium is $B$ cell dependent. J Immunol 2001;167:4710-18.

6 Zhang Z, Bridges SL. Pathogenesis of rheumatoid arthritis. Role of B lymphocytes. Rheum Dis Clin North Am 2001;27:335-53.

7 Dörner T, Burmester GR. The role of $B$ cells in rheumatoid arthritis: mechanisms and therapeutic targets. Curr Opin Rheumatol 2003; 15:246-52

8 Shiokawa S, Matsumoto N, Nisimura J. Clonal analysis of B cells in the synovial membrane of patients with rheumatoid arthritis. Scand J Rheumatol 2003:32:12-18.

9 Tighe H, Carson DA. Rheumatoid factors. In: Kelly WN, Rudy S, Sledge CD, Harris ED eds. Textbook of rheumatology. Philadelphia: Saunders, 1997:241-9.

10 Van Zeben D, Hazes JMW, Zwinderman AH, Cats A, van der Voort EA, Breedveld FC. Clinical significance of rheumatoid factors in early rheumatoid arthritis: results of a follow-up study. Ann Rheum Dis 1992; $51: 1029-35$

11 McLaughlin P, Grillo-Lopez AJ, Link BK, Czuczman MS, Williams ME, Heyman MR, et al. Rituximab chimeric anti-CD20 monoclonal antibody therapy for relapsed indolent lymphoma: half of patients respond to a four-dose treatment program. J Clin Oncol 1998;16:2825-33.

12 Anderson DR, Grillo-Lopez AJ, Varns C, Chambers KS, Hanna N Targeted anti-cancer therapy using rituximab, a chimeric anti CD-20 antibody (IDEC-C2B8) in the treatment of non-Hodgkin's B-cell lymphoma. Biochem Soc Trans 1997:25:705-8.

13 Clynes RA, Towers TL, Presta LG, Ravetch JV. Inhibitory Fc receptors modulate in vivo cytotoxicity against tumour targets. Nat $\mathrm{Med}$ 2000;6:443-6

14 Reff ME, Carner K, Chambers KS, Chinn PC, Leonard JE, Raab R, et al. Depletion of $B$ cells in vivo by chimeric mouse human monoclonal antibody to CD20. Blood 1994;83:435-45.

15 McLaughlin P, Hagemeister FB, Grillo-Lopez AJ. Rituximab in indolent lymphoma: the single agent pivotal trial. Semin Oncol 1999;26:79-87.

16 Hainsworth JD, Litchy S, Burris HA, Scullin DC, Corso SW, Yardley DA et al. Rituximab as first-line and maintenance therapy for patients with indolent non-Hodgkin's lymphoma. J Clin Oncol 2002;20:4261-7.

17 Protheroe A, Edwards JCW, Simmons A, Maclennan K, Selby P. Remission of inflammatory arthropathy in association with anti-CD20 therapy for non-Hodgkin's lymphoma. Rheumatology (Oxford) 1999;38:1150-2.

18 Edwards JCW, Cambridge G. Sustained improvement in rheumatoid arthritis following a protocol designed to deplete B lymphocytes. Rheumatology (Oxford) 2001;40:205-1 1 .

19 Czuczman MS. CHOP plus rituximab chemoimmunotherapy of indolent B cell lymphoma. Semin Oncol 1999;26:88-96.

20 Edwards JCW, Szczepanski L, Szechinski J, Filipowicz-Sosnowska A Close D, Stevens RM, et al. Efficacy and safety of rituximab, a B-cell targeted chimeric monoclonal antibody: a randomized, placebo controlled trial in patients with rheumatoid arthritis [abstract]. Arthritis Rheum 2002;46(suppl):S197. 
21 De Vita S, Zaja F, Sacco S, De Candia A, Fanin R, Ferraccioli G Efficacy of selective $B$ cell blockade in the treatment of rheumatoid arthritis. Evidence for a pathogenic role of B cells. Arthritis Rheum 2002;46:2029-33.

22 Leandro MJ, Edwards JCW, Cambridge G. Clinical outcome in 22 patients with rheumatoid arthritis treated with $B$ lymphocyte depletion. Ann Rheum Dis 2002;61:883-8.

23 Tuscano JM. Successful treatment of infliximab-refractory rheumatoid arthritis with rituximab. Arthritis Rheum 2002;46:3420, LB 11

24 Stasi R, Pagano A, Stipa E, Amadori S. Rituximab chimeric anti-CD20 monoclonal antibody treatment for adults with chronic idiopathic thrombocytopenic purpura. Blood $2001 ; 98: 952-7$.

25 Zaja F, De Vita S, Mazzaro C, Sacco S, Damiani D, De Marchi G, et al., Efficacy and safety of rituximab in type II mixed cryoglobulinemia. Blood 2003;101:3827-34.
26 Pestronk A, Florence J, Miller T, Choksi R, Al-Lozi MT, Levine TD.

Treatment of IgM antibody associated polyneuropathies using rituximab. J Neurol Neurosurg Psychiatry 2003;74:485-9.

27 Lipsky PE. Systemic lupus erythematosus: an autoimmune disease of B cell hyperactivity. Nat Immunol 2001;2:764-6.

28 Anolik JH, Campbell D, Felgar R, et al. B lymphocyte depletion in the treatment of systemic lupus (SLE): phase I/II trial of rituximab (Rituxan ${ }^{\circledR}$ ) in SLE [abstract]. Arthritis Rheum 2002;46(suppl):S289.

29 Leandro MJ, Edwards JC, Cambridge G, Ehrenstein MR, Isenberg DA. An open study of $B$ lymphocyte depletion in systemic lupus erythematosus. Arthritis Rheum 2002:46:2673-7.

30 Edwards JCW, Leandro M, Cambridge G. B-lymphocyte depletion therapy in rheumatoid arthritis and other autoimmune disorders. Biochem Soc Trans 2002;30:824-8.

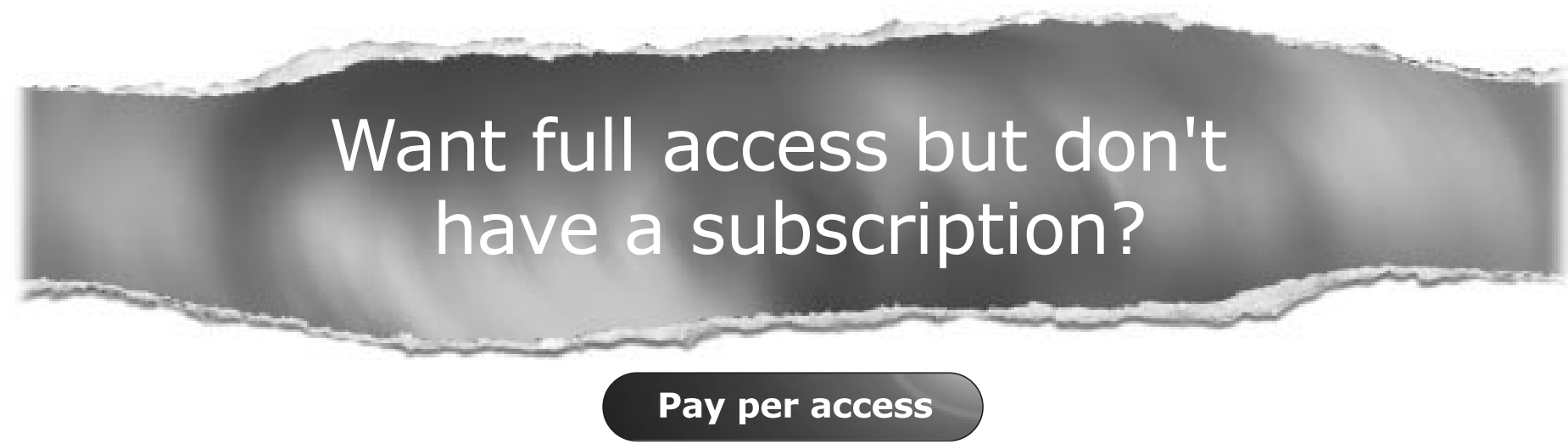

For just US $\$ 25$ you can have instant access to the whole website for 30 days. During this time you will be able to access the full text for all issues (including supplements) available. You will also be able to download and print any relevant pdf files for personal use, and take advantage of all the special features Annals of the Rheumatic Diseases online has to offer.

www.annrheumdis.com 